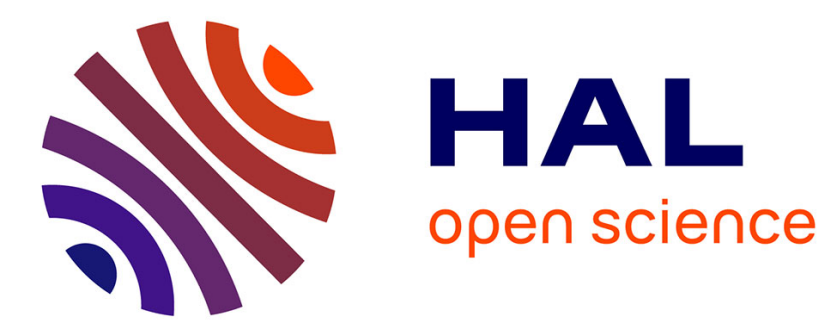

\title{
Enhancing accuracy of low-dropout regulator susceptibility extraction with on-chip sensors
}

Jian-Fei Wu, Etienne Sicard, Alexandre Boyer, Sonia Ben Dhia, Jiancheng Li, Rongjun Shen

\section{- To cite this version:}

Jian-Fei Wu, Etienne Sicard, Alexandre Boyer, Sonia Ben Dhia, Jiancheng Li, et al.. Enhancing accuracy of low-dropout regulator susceptibility extraction with on-chip sensors. Electronics Letters, 2012, 48 (11), pp.649-650. 10.1049/el.2012.0407 . hal-00709287

\section{HAL Id: hal-00709287 \\ https://hal.science/hal-00709287}

Submitted on 25 Jun 2012

HAL is a multi-disciplinary open access archive for the deposit and dissemination of scientific research documents, whether they are published or not. The documents may come from teaching and research institutions in France or abroad, or from public or private research centers.
L'archive ouverte pluridisciplinaire HAL, est destinée au dépôt et à la diffusion de documents scientifiques de niveau recherche, publiés ou non, émanant des établissements d'enseignement et de recherche français ou étrangers, des laboratoires publics ou privés. 


\section{Enhancing accuracy of low-dropout regulator susceptibility extraction with on-chip sensors}

J.F. Wu, E. Sicard, A. Boyer, S. Ben Dhia, J.C. Li and R.J. Shen

Presented is a new test method that consists in monitoring on-chip internal voltages during susceptibility tests of integrated circuits. An on-chip sensor was installed at several internal nodes within lowdropout regulators to measure the distortion of internal signals induced by the coupling of electromagnetic interference. The comparison between external and internal measurement results shows that onchip sensor techniques enhance the extraction of circuit susceptibility levels, especially at high frequencies.

Introduction: Owing to the increasing complexity of electromagnetic environments, concerns about the susceptibility of low-dropout (LDO) regulators are becoming ever more important, especially in critical embedded electronic systems for automotive or aerospace applications [1], the performances of which are directly linked to power supply voltage integrity. Recently, various papers have described the failure mechanisms of LDO regulators under conducted electromagnetic interference (EMI), linked to operational amplifiers (opamps) [2] or bandgap cells [3]. In these papers, measurements of susceptibility were performed with external tools, such as oscilloscopes and power meters. This Letter proposes the use of on-chip measurement methods described in [4], which give more efficient results by avoiding the effects of parasitic capacitors and inductors associated with bonding, package leads, printed circuit boards (PCBs) and measurement probes that filter the actual phenomena. This Letter presents a study of the conducted susceptibility of an integrated LDO regulator module. The comparison between external and internal measurements shows differences that indicate that only on-chip sensors are able to measure the actual susceptibility level.

Description of test chip and DPI measurement setup: The LDO regulator under test is part of a test chip designed using the Freescale CMOS $90 \mathrm{~nm}$ process. Its purpose was to provide a regulated power supply voltage to a small digital core. The most prominent feature of the chip is the presence of on-chip voltage sensors on several internal nodes, working as frequency aliasing sub-samplers [4]. High-frequency signals can be measured (up to $10 \mathrm{GHz}$ ) with relaxed constraints on hardware bandwidth requirements.

Fig. 1 shows the structure of the voltage regulator module and the basic setup for EMI study. The regulator mainly includes a Kuijk bandgap voltage reference with startup circuit [6] and an output feedback amplifier. Harmonic disturbances from 1 to $2000 \mathrm{MHz}$ are coupled to the $V_{\mathrm{IN}}$ power line according to the direct power injection (DPI) standard [5]. Two on-chip sensors were used in this module to monitor bandgap and regulator output voltages inside the chip. Each sensor uses a separate power supply and substrate isolation techniques to prevent conducted disturbances from affecting its operation.

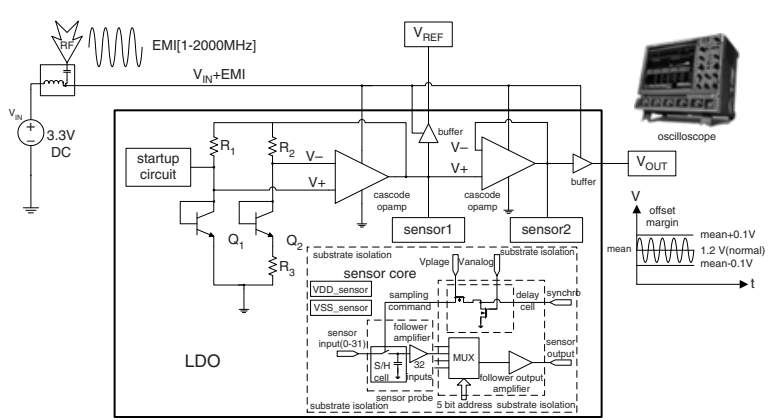

Fig. 1 Test chip structure and general setup for DPI measurement

LDO failure types and mechanism analysis: When EMI is superimposed on the power supply pin, regulator and bandgap outputs are distorted. Two different failure types arise during conducted susceptibility tests carried out on the power supply pin: first, a part of the incoming disturbance spreads to the output signal; and secondly, a
DC offset is generated by a parasitic rectification effect intrinsic to the circuit under test. The offset can be positive or negative depending on EMI frequency and amplitude. The first type of distortion can be eliminated by filtering, but the second type cannot and degrades the regulation performance of the LDO. A uniform criterion is defined to analyse the susceptibility, which consists in setting a margin of $\pm 100 \mathrm{mV}$ on the offset measured on the regulator output. Fig. 2 shows examples of on-chip sensor 2 measurements for two different RFI frequencies ( $700 \mathrm{MHz}$ and $1 \mathrm{GHz}$ ).

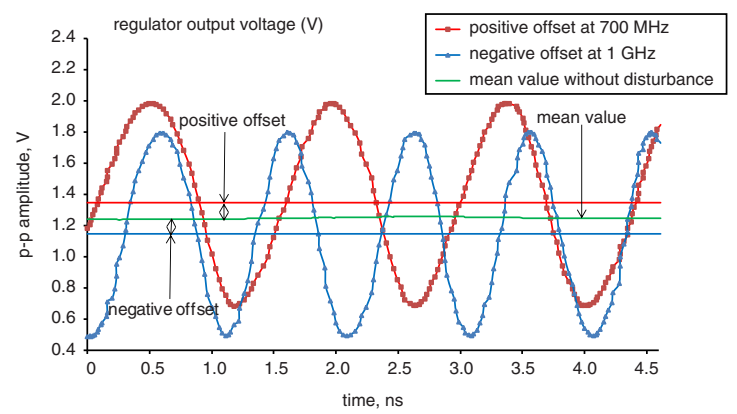

Fig. 2 Internal failure monitored by on-chip sensor at $700 \mathrm{MHz}$ and $1 \mathrm{GHz}$

On-chip sensor measurements and comparison with external methods: The acquisition is based on an on-chip sample-and-hold circuit that directly probes the voltage within integrated circuit (IC) interconnects. An associated post-processing phase reconstructs the timing waveform. The detailed principle of this on-chip sensor is given in [7].

With the on-chip sensor, we can use the advantage of inner sampling and enhance our measurement of the actual susceptibility level in the chip, as shown in Figs. $3 a$ and $b$. First, Fig. $3 a$ shows the susceptibility level of the regulator to conducted harmonic disturbance applied on the $V_{\text {IN }}$ pin measured according to two methods: the EMI-induced offset is measured on the regulator output either externally (after output buffer and package) with an oscilloscope or internally with the on-chip sensor 2. When failures arise $( \pm 100 \mathrm{mV}$ offset), the two types of measurement give different results between $700 \mathrm{MHz}$ and $1 \mathrm{GHz}$, indicating different susceptibility levels of the regulator.

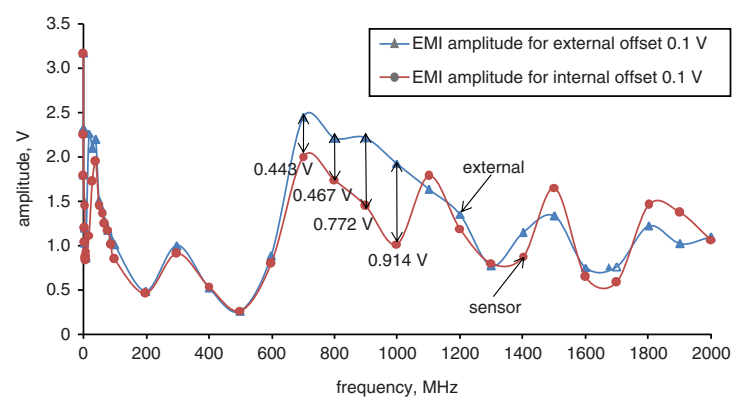

a

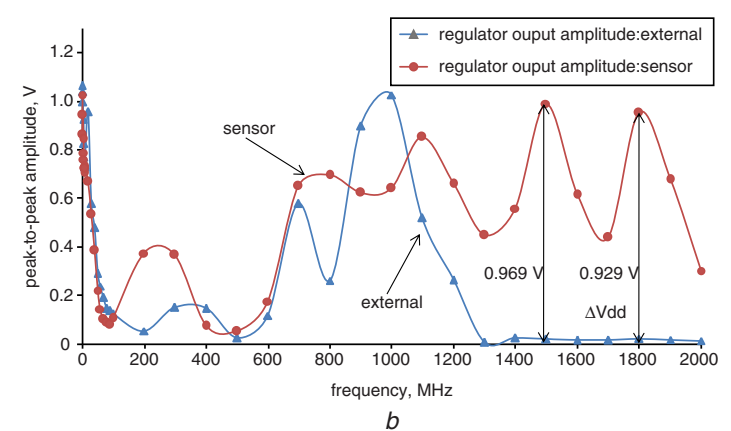

Fig. 3 Comparison between external and on-chip sensor sampling methods $a$ Internal and external voltage fluctuation measured on regulator output pin to obtain $+100 \mathrm{mV}$ offset

$b$ Fluctuation measured in amplitude of regulator output voltage under same offset criterion

The characterisation of the harmonic distortion of the regulator output is presented in Fig. $3 b$. The amplitude of regulator output voltage is 
measured externally and internally when a failure is detected. The two types of measurement give different results above $600 \mathrm{MHz}$. It can be seen that, above $1.3 \mathrm{GHz}$, the amplitude of the regulator output measured externally decreases to almost $0 \mathrm{~V}$ while the on-chip sensor gives a totally different result. It indicates a voltage fluctuation of several hundred millivolts and thus gives the actual level of harmonic distortion affecting the regulator output. The differences between internal and external measurements of regulator output distortion presented in Figs. $3 a$ and $b$ can be explained by the contribution of bonding, package, $\mathrm{PCB}$ and $\mathrm{I} / \mathrm{O}$ output buffer structures. Above several hundred megahertz, they act as a lowpass filter, which reduces or even eliminates the disturbance. External measurement methods cannot give the actual amount of degradation of output signal and thus an efficient diagnosis of the electromagnetic susceptibility of an integrated regulator. An on-chip sensor constitutes an accurate measurement method of the susceptibility of each internal block of an IC, such as an integrated LDO regulator.

Conclusions: This Letter has highlighted the difference between external and internal measurement and draws attention to accuracy issues of external measurements of an integrated low-dropout regulator. With the same failure criterion, sensor measurements indicate lower EMI amplitude, thus invalidating the LDO regulator within the range 700-1000 MHz. Moreover, above $1 \mathrm{GHz}$ the on-chip sensor detects a larger harmonic distortion level of the regulator output than external measurement. External measurement methods underestimate the susceptibility level and the disturbance of the regulator. On-chip measurements constitute an efficient technique for addressing susceptibility issues in critical internal blocks within system-on-chip, systemin-package or $3 \mathrm{D}$ circuits.

Acknowledgments: The authors acknowledge the full support of the China Scholarship Council and thank C. Lemoine from INSA, University of Toulouse, France, for software support.
(C) The Institution of Engineering and Technology 2012

8 February 2012

doi: $10.1049 / \mathrm{el} .2012 .0407$

One or more of the Figures in this Letter are available in colour online.

J.F. Wu, J.C. Li and R.J. Shen (School of Electronic Science and Engineering, NUDT, Changsha, Hunan 410073, People's Republic of China)

E-mail: wujianfei990243@gmail.com

E. Sicard (INSA, University of Toulouse, 135 av. de Rangueil, Toulouse 31077, France)

A. Boyer and S. Ben Dhia (LAAS-CNRS, 7 avenue du colonel Roche, Toulouse 31077, France)

\section{References}

1 Ben Dhia, S., Ramdani, M., and Sicard, E.: 'Electromagnetic compatibility of integrated circuits: techniques for low emission and susceptibility' (Springer, USA, 2006, ISBN: 0-387-26600-3)

2 Fiori, F., and Crovetti, P.S.: 'Linear voltage regulator susceptibility to conducted EMI', Ind. Electron., 2002, 4, pp. 1398-1403

3 Orietti, E., Montemezzo, N., Buso, S., Meneghesso, G., Neviani, A., and Spiazzi, G.: 'Reducing the EMI susceptibility of a Kuijk bandgap', IEEE Trans. Electromagn. Compat., 2008, 50, (4), pp. 876-886

4 Vrignon, B., and Ben Dhia, S.: 'On-chip sampling sensors for high frequency signals measurement: evolution and improvements'. Proc. 5th IEEE Int. Caracas Conf. on Devices, Circuits and Systems, Punta Cana, Dominican Republic, November 2004

5 IEC 623132-4: Integrated Circuits, Measurement of Electromagnetic Immunity - Part 4: Direct RF Power Injection Method

6 Kuijk, K.E.: 'A precision reference voltage source', IEEE J. Solid-State Circuits, 1973, 8, (3), pp. 222-226

7 Ben Dhia, S., Boyer, A., Vrignon, B., and Deobarro, M.: 'IC immunity modeling process validation using on-chip measurements'. 2011 12th Latin American Test Workshop (LATW), Porto de Galinhas, Brazil, March 2011, pp. 1-6 